\title{
Hybrid guidewires: Analysis and comparison of the mechanical properties and safety profiles
}

Bryan D Hinck ${ }^{1}$; Anthony S. Emmott ${ }^{2}$; Mohamed Omar ${ }^{3}$; Sarah Tarplin ${ }^{1}$; Ben H. Chew ${ }^{2,4}$; Manoj Monga ${ }^{1,4}$

${ }^{1}$ Glickman Urologic and Kidney Institute, Cleveland Clinic Foundation, Cleveland, OH, United States; ${ }^{2}$ Department of Urologic Sciences, University of British Columbia, Vancouver, BC, Canada; ${ }^{3}$ Urology Department, Menoufia University, Egypt; ${ }^{4}$ Endourologic Disease Group for Excellence (EDGE) Research Consortium

Funding: Cook Urological provided financial support for this study.

Cite as: Can Urol Assoc J 2018 July 31; Epub ahead of print. http://dx.doi.org/10.5489/cuaj.5396

Published online July 31, 2018

$* * *$

\section{Abstract}

Introduction: Hybrid guidewires are commonly used in urology due to the advantage of an atraumatic hydrophilic tip, which facilitates negotiating tight areas, coupled with an unkinkable nitinol core shaft that is easy to work over due to the teflon coating. Our aim was to compare the physical and mechanical properties of five commercially available hybrid guidewires to assess their characteristics and functionality.

Methods: In vitro testing was performed on the following straight-tipped 0.035 inch guidewires: Sensor (Boston Scientific), Solo Plus (Bard), UltraTrack (Olympus), Rio Tracer (Rocamed), and Motion (Cook). We evaluated characteristics impacting function (tip flexibility, shaft stiffness, lubricity) and safety (perforation force). Measurements included tip flexibility, lubricity, shaft buckling, and force required to perforate a sheet of aluminum foil.

Results: The Motion had the highest tip bending force $(\mathrm{p}<0.00001)$. The Rio Tracer had the stiffest shaft $(\mathrm{p}<0.00001)$, followed by the Solo Plus and the Motion, which were significantly stiffer than the Sensor and UltraTrack $(\mathrm{p}<0.00001)$. The Solo Plus and UltraTrack had the greatest perforation force ( $\mathrm{p}=0.00023$ ), and the Rio Tracer had the lowest perforation force $(p=0.016)$ when compared to the Sensor. There was no significant difference in frictional force between the five guidewires $(\mathrm{p}=0.1516)$.

Conclusions: The Solo Plus and UltraTrack required the greatest force to perforate, which conveys a higher safety margin. The RioTracer is the stiffest guidewire, which may be beneficial for instrument insertion with the tradeoff of having a lower perforation force. The clinical 
significance of higher tip-bending forces (unfavourable) and higher shaft-bending forces (favourable) deserve further investigation.

\section{Introduction}

Guidewires have been a mainstay of endovascular procedures and are now a fundamental tool used in endourological procedures for establishing access in the urinary system. ${ }^{1}$ Numerous manufacturers have produced guidewires with a variety of materials, coatings, flexibility, length, diameters and other mechanical properties for suitability in a wide range of clinical scenarios. The ideal wire in a general setting requires a combination of tip flexibility and hydrophilicity, as well as shaft stiffness and retention friction. Tip flexibility is required to maneuver past obstructions without perforation whereas stiffness allows manipulation of instruments, access sheaths and stents without kinking. Rigidity is also important for smooth transmission of torque from the end of the guidewire to the tip. Hydrophilicity at the tip is beneficial for ease of guidewire insertion, however sufficient friction of the wire shaft is necessary to avoid loss of wire position in the urinary tract.

The combination of these factors determines the usability and safety of each guidewire depending on the clinical application. Hybrid guidewires have been developed to maximize the benefit of a hydrophilic, soft tip with a rigid shaft that has sufficient friction to maintain wire position. The Sensor (Boston Scientific) was the first hybrid guidewire introduced commercially. Since the introduction of the Sensor wire in 2000, other hybrid guidewires have not entered the market until recently in the last few years including the Solo Plus (Bard), UltraTrack (Olympus), Rio Tracer (Rocamed) and Motion (Cook). Our specific aim was to compare the physical and mechanical properties of these five commercially available hybrid guidewires to assess their characteristics, safety and functionality to aid in clinical decision making for urologists.

The factors we evaluated to test clinical suitability included tip flexibility, shaft stiffness, lubricity and perforation force. Tip flexibility was measured to inform the action of a wire around an obstruction in the urinary tract. The force required for perforation by the tip of the wire was used to evaluate wire safety with respect to risk of tissue perforation. Shaft stiffness was assessed to determine the predicted level of bending or kinking that may be experienced upon insertion of a wire or during manipulation of instruments over the wire. Lubricity of the wires was calculated as an indicator of likelihood for wire slippage from the urinary tract with the caveat that higher friction forces may impair insertion of the wire or impede insertion of devices over the wire.

\section{Methods}

In vitro testing was performed on the straight-tipped 0.035 inch: Sensor (Boston Scientific), Solo Plus (Bard), UltraTrack (Olympus), Rio Tracer (Rocamed), and Motion (Cook) wires. We evaluated characteristics impacting function (tip flexibility, shaft stiffness, and lubricity) and safety (perforation force), as previously described by our group. ${ }^{2}$ 
A load cell (Series MR03-2, Mark-10, Copiague, NY) attached to a linear motion stage with a stepper motor was used to measure forces. The motorized stage advanced the wire at a constant speed of $5.0 \mathrm{~cm} / \mathrm{min}$.

Perforation force

Perforation force was evaluated by stabilizing each wire in the dilator of a ureteral access sheath to prevent wire bending and securing the wire to the force gauge (Figure 1). The access sheath was placed flush to the aluminum foil $(0.016 \mathrm{~mm}$ thick) which was secured between two washers. The maximal force required to puncture the foil was then recorded with 10 trials of each wire.

\section{Tip bending}

Tip flexibility was determined by isolating the distal $5 \mathrm{~cm}$ portion of each guidewire and measuring the peak force required to compress a distance $3.0 \mathrm{~cm}$ while securing the tip in a small hole in a wooden block (Figure 2). This allowed for testing of the hydrophilic portion in isolation from the stiffer shaft. A total of 20 trials were conducted for each wire.

\section{Shaft bending}

Shaft stiffness was determined by measuring the peak force to compress a $30 \mathrm{~cm}$ section of the shaft a distance of $10 \mathrm{~cm}$. The wire was fixed at a point $30 \mathrm{~cm}$ from the linear motion stage and this distance was closed to $20 \mathrm{~cm}$ at a speed of $5.0 \mathrm{~cm} / \mathrm{min}$ (Figure 3). 10 trials were conducted on each wire with a new segment of wire used for each trial.

Frictional force

Lubricity was measured by extracting each wire through simulated tissue (sliced bologna at $22^{\circ} \mathrm{C}$ ) at a constant speed of $5.0 \mathrm{~cm} / \mathrm{min}$ for $15 \mathrm{~cm}$ (Figure 4). The continuous force was measured and the average force over a 5 second period was recorded. A total of 10 trials were conducted for each wire.

\section{Statistical analysis}

Single-factor analysis of variance (ANOVA) was used to determine the statistical difference among all guidewires for each test. The Student $\mathrm{t}$ test was used for pairwise comparisons between the guidewires within each test. Significance was set at $P<0.05$.

\section{Results}

The Motion wire had the highest tip bending force (Table 1, Figure 5, $\mathrm{p}<0.00001$ ). The Rio Tracer wire had the stiffest shaft (ANOVA $\mathrm{p}<0.00001$ ), followed by the Solo Plus and the Motion which were significantly stiffer than the Sensor and UltraTrack (ANOVA $\mathrm{p}<0.00001$ ). There was no difference in stiffness between the Solo Plus and the Motion or the Sensor and UltraTrack when compared head to head (t-test $\mathrm{p}=0.6925$ and $\mathrm{p}=0.2180$ respectively). The Solo Plus and UltraTrack wires had the greatest perforation force (ANOVA $\mathrm{p}=0.00023$ ) with no significant difference between the two (t-test $\mathrm{p}=0.785$ ), and the Rio Tracer wire had the lowest perforation force (t-test $\mathrm{p}=0.016$ ) when compared to the Sensor. There was no significant difference between the 5 wires in regards to frictional force (ANOVA $\mathrm{p}=0.1516$ ). 


\section{Discussion}

Guidewires ultimately serve two main purposes: to provide access to the urinary tract and to function as a guide for placement of stents and other instruments. ${ }^{3}$ These tasks require different mechanical and functional characteristics of guidewires. In order to provide access to the urinary tract, tip flexibility and minimal friction promote easy passage of the guidewire into the urinary tract. ${ }^{4}$ For placement of stents, catheters or ureteral access sheaths over the guidewire, the shaft stiffness is most relevant. ${ }^{3}$ In addition to these functional characteristics, one must also consider the safety aspect to minimize the risk of ureteral perforation.

A combination of characteristics will lead to a more desirable wire. Greater tip flexibility (ie. lower tip bending force) may improve the ability of the wire to maneuver around an obstructing stone in the ureter since it is thought the tip will bend more easily to negotiate tortuous anatomy. The Motion wire required greater tip bending force than the other four wires. This wire is more likely to remain straight before bending as it negotiates tight anatomy which may impact its ability to bypass an obstruction. Based on this bench data, the other 4 wires tested may be a better option when aiming to maneuver around an obstructing stone or tortuous ureter.

Lubricity did not differ significantly between these wires. All five guidewires tested had similar dynamic frictional forces, suggesting that there would be no difference in terms of lubricity when inserting the guidewires into the urinary tract. This is likely a result of all five wires utilizing polytetrafluoroethylne (PTFE or teflon) as the material of choice for the coating. Lubricity should therefore not be a significant consideration when deciding on a wire to use between those tested.

The material of the guidewire core plays a role in the stiffness of the shaft, with stainless steel and shape memory alloys such as Nitinol being the most commonly used materials. ${ }^{5}$ Nitinol is a nickel and titanium alloy benefiting from a kink-resistant shaft with a potential drawback of less stiffness compared to stainless steel. All wires tested in this study were composed of a shape memory alloy, including Nitinol for the Sensor, UltraTrack, Rio Tracer and Motion wires whereas the Solo Plus utilized a proprietary core named Triton, which contains cobalt and is reported to confer increased stiffness. Stiffness is thought to enhance the ability of the guidewire to facilitate passage of instruments such as a ureteral access sheath. Furthermore, increased stiffness may also help translate movements from outside the body resulting in better control of tip of the wire during manipulation. Stiffness was similar among 4 of the wires with the Rio Tracer being the stiffest which may facilitate passage of a UAS without kinking or buckling. However, the tradeoff to the Rio Tracer is that it requires the lowest force to cause perforation which is an important safety consideration when negotiating an impacted stone. This stiffness of the shaft may be partly responsible for the lower force required for the tip to perforate in our testing model. While direct comparison of shaft stiffness between the Rio Tracer and the Amplatz SuperStiff non-hybrid guidewire from a previous study is not possible, these two wires may have comparable stiffness. ${ }^{2}$ Further direct comparison of these two wires would be beneficial as the Rio Tracer would have the added benefit of increased kink-resistance over a 
stainless steel wire. The Solo Plus, with Triton core, and Motion, with Nitinol core, were found to have an intermediate level of shaft stiffness which could provide a balance of shaft stiffness with protective greater perforation forces. The least stiff were the Nitinol-based Sensor and UltraTrack wires, although only marginally less than the Solo Plus and Motion wires.

A soft tip is desirable to help negotiate tortuous anatomy or impacted stones and we tested 2 characteristics for the tip: the force required to bend the tip which was greatest in the Motion wire and similar among the other 4 wires and the force to perforate a sheet of aluminum foil. The desirable quality of a tip is to require a higher force to perforate-i.e. you would have to push more firmly to cause a perforation. The Rio Tracer wire perforated at the lowest force level which in itself is not desirable. The Solo Plus and UltraTrack wires required greater forces to perforate than the Motion or Sensor wires wire. The Sensor and UltraTrack had the lowest shaft stiffness of the group, with the UltraTrack having a greater perforation force than the Sensor wire. In this regard, the Solo Plus and UltraTrack provide the lowest risk followed by the Motion and Sensor wire for perforation and if safety is the main concern in a particular clinical scenario these would be the recommended guidewires.

Comparison of the Sensor guidewire between this study and a previous study show different absolute values for the same characteristics tested, which is likely a result of calibration differences due to small variations in equipment setup. ${ }^{2}$ We attempted to minimize error by utilizing the same equipment setup for each measurement, having a single operator perform the testing and by repeating an internal control with each guidewire to confirm calibration. This allowed for relative comparison of the guidewires tested, however comparison to previous values measured with different experimental calibrations is limited.

There are certain limitations to the mechanical testing performed in this study. We present a comparison of important functional characteristics which are thought to influence how these guidewires perform in a clinical setting. However, we have not taken into account how they may perform in live tissue. Although aluminum foil and bologna have been used as models for ureteral tissue previous to this study, ${ }^{3,4}$ these wires may perform differently when used in real-world situations. Additionally, each characteristic has been tested in isolation. Future studies could focus on evaluating performance of guidewires based on a combination of characteristics such as tip flexibility plus shaft stiffness as the wires may differ at the transitional segment between rigid staff and flexible tip. Our testing apparatus for perforation force is heavily dependent on the stiffness of the shaft since this is translated to the tip of the wire. Nonetheless, the information presented will certainly be of great benefit to urologists in selecting the best product for their clinical tasks.

\section{Conclusion}

The Bard Solo Plus and Olympus UltraTrack wires required the greatest force to perforate (followed closely by the Boston Scientific Sensor and Cook Motion) which conveys a protective safety margin. The Rocamed RioTracer has the stiffest shaft which may be beneficial for instrument insertion, but may translate this stiffness to the tip resulting in a lower perforation force in our testing, and thus a lower margin of safety. The Cook Motion had the highest tip 
bending force which may make it more difficult to maneuver past obstructing stones compared to the other wires tested. The five guidewires did not differ in terms of lubricity. Our study evaluated each property in isolation and the clinical significance of these properties would require testing each wire's properties as a whole; nevertheless, these individual properties will help each urologist determine which wire may be utilized in specific situations. 


\section{References}

1. Fritzsche P, Moorhead JD, Axford PD et al. Urologic applications of angiographic guide wire and catheter techniques. J Urol 1981;125:774-80.

2. Sarkissian C, Korman E, Hendlin K et al. Systematic evaluation of hybrid guidewires: shaft stiffness, lubricity, and tip configuration. Urology 2012;79:513-7.

3. Clayman M, Uribe CA, Eichel L et al. Comparison of guide wires in urology. Which, when and why? J Urol 2004;171:2146-50.

4. Torricelli FC, De S, Sarkissian C et al. Hydrophilic guidewires: evaluation and comparison of their properties and safety. Urology 2013;82:1182-6.

5. Liguori G, Antoniolli F, Trombetta C et al. Comparative experimental evaluation of guidewire use in urology. Urology 2008;72:286-9. 


\section{Figures and Tables}

Fig. 1. Testing of perforation force, evaluated by measuring the maximal force required to puncture aluminum foil (0.016 mm thick).

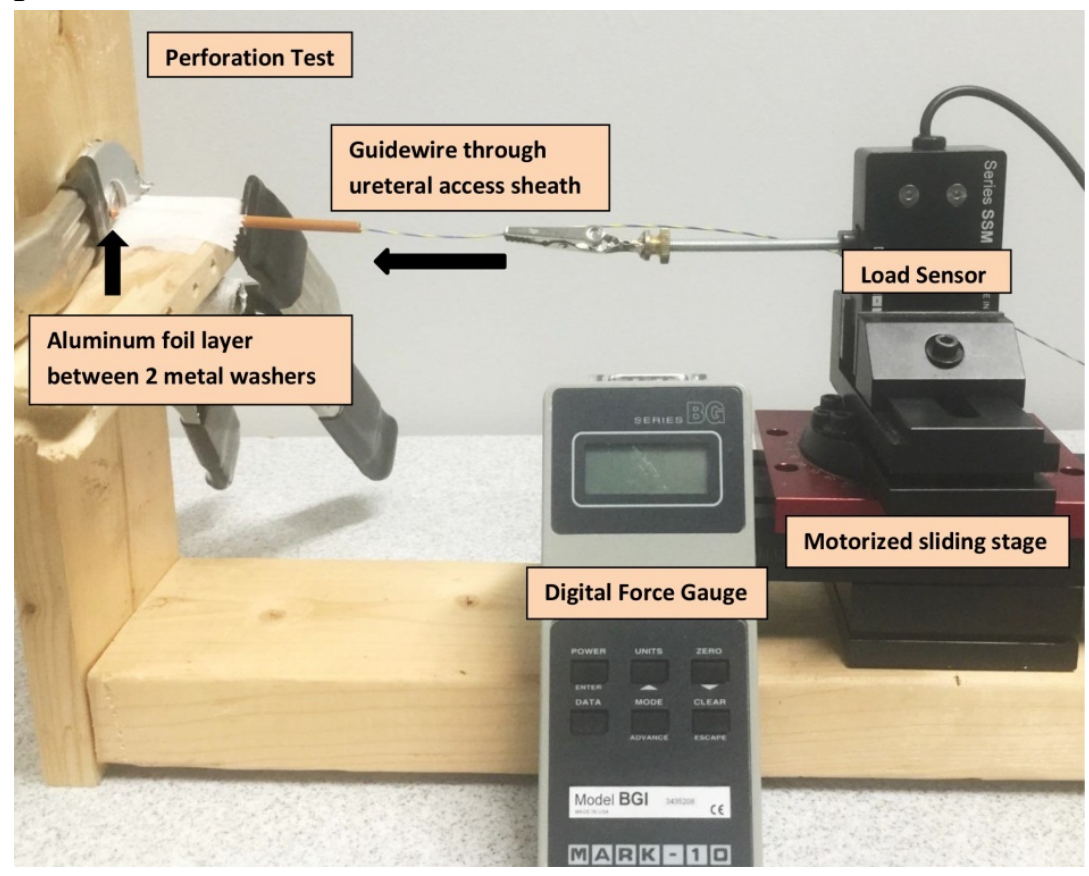

Fig. 2. Testing of tip flexibility, determined by measuring the peak force required to compress the distal $5 \mathrm{~cm}$ portion of each guidewire a distance of $3.0 \mathrm{~cm}$.

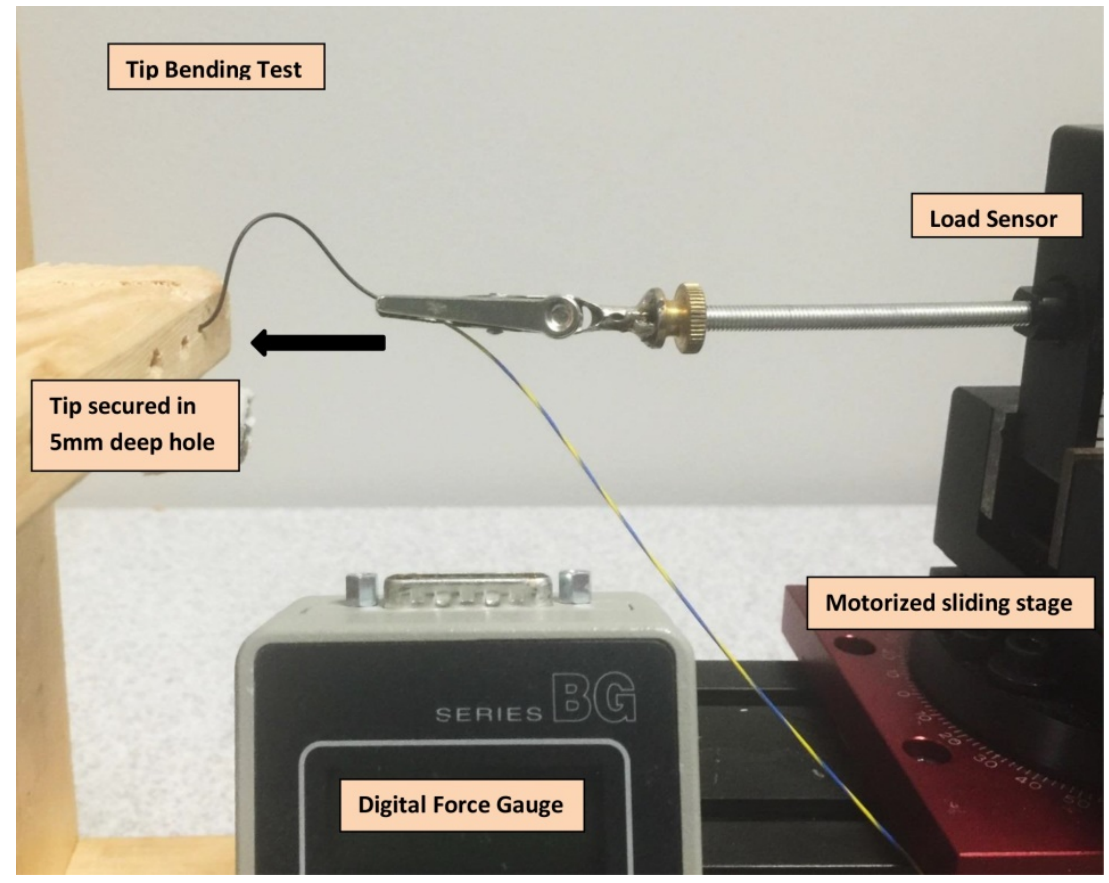


Fig. 3. Testing of shaft stiffness, determined by measuring the peak force to compress a $30 \mathrm{~cm}$ section of the shaft a distance of $10 \mathrm{~cm}$ at a speed of $5.0 \mathrm{~cm} / \mathrm{min}$.

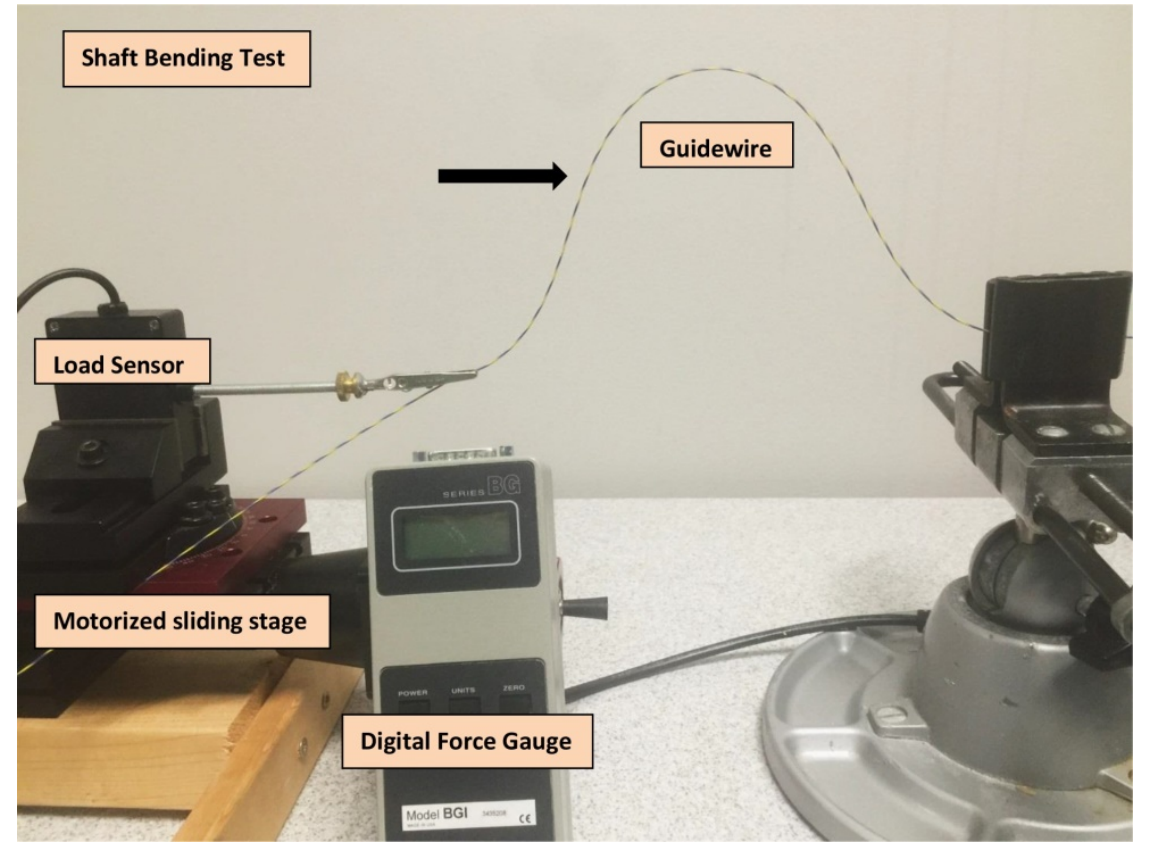

Fig. 4. Testing of dynamic frictional force (lubricity), measured by extracting guidewire through simulated tissue at a constant speed of $5.0 \mathrm{~cm} / \mathrm{min}$ for $15 \mathrm{~cm}$.

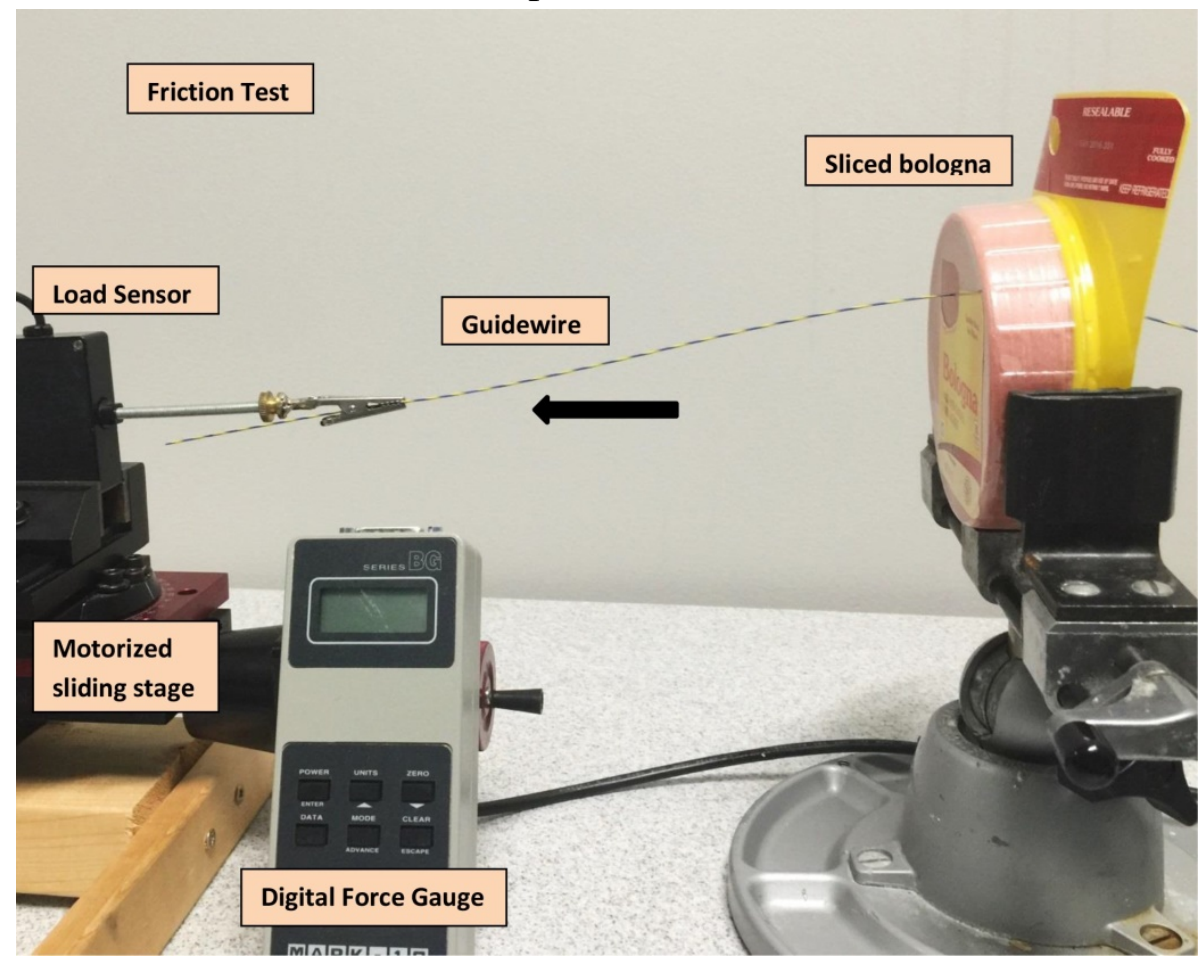


Fig. 5. (A) Average force required for guidewire tip to perforate aluminum foil \pm 1 standard deviation (SD). (B) Average force required to bend tip $3 \mathrm{~cm} \pm 1$ SD. (C) Average force required to bend shaft $10 \mathrm{~cm} \pm 1 \mathrm{SD}$. (D) Average dynamic frictional force $\pm 1 \mathrm{SD}$.

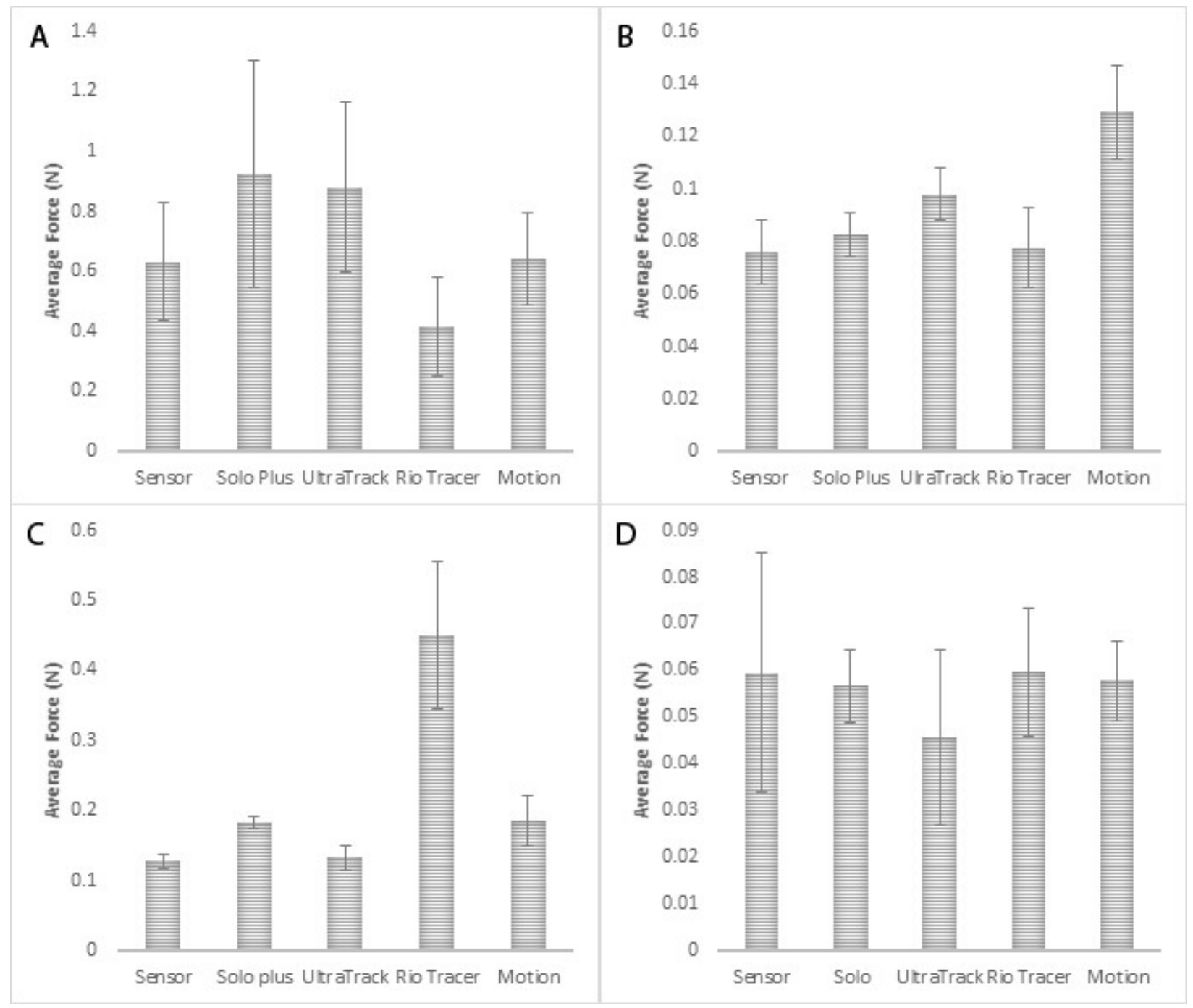




\begin{tabular}{|l|l|c|c|c|c|c|}
\hline $\begin{array}{l}\text { Table 1. Comparison of guidewires with values for perforation, tip bending, shaft bending and } \\
\text { frictional forces (Newtons) }\end{array}$ & Sensor & Solo Plus & UltraTrack & Rio Tracer & Motion & ANOVA \\
\hline Forces & $0.633 \pm 0.196$ & $0.923 \pm 0.378$ & $0.881 \pm 0.286$ & $0.416 \pm 0.167$ & $0.641 \pm 0.154$ & $\mathrm{p}=0.00023$ \\
\hline Perforation & $0.076 \pm 0.012$ & $0.082 \pm 0.008$ & $0.098 \pm 0.010$ & $0.077 \pm 0.015$ & $0.129 \pm 0.018$ & $\mathrm{p}<0.00001$ \\
\hline Tip bending & $0.128 \pm 0.009$ & $0.183 \pm 0.008$ & $0.133 \pm 0.017$ & $0.451 \pm 0.105$ & $0.185 \pm 0.035$ & $\mathrm{p}<0.00001$ \\
\hline $\begin{array}{l}\text { Shaft } \\
\text { bending }\end{array}$ & 0.105 & & & & \\
\hline Frictional & $0.059 \pm 0.026$ & $0.056 \pm 0.008$ & $0.046 \pm 0.019$ & $0.060 \pm 0.014$ & $0.058 \pm 0.008$ & $\mathrm{p}=0.15159$ \\
\hline
\end{tabular}

